\title{
Chinese homozygous Machado-Joseph disease (MJD)/SCA3: a case report
}

\author{
Sheng Zeng ${ }^{1}$, Junsheng Zeng ${ }^{1}$, Miao He ${ }^{1}$, Xianfeng Zeng ${ }^{1}$, Yao Zhou ${ }^{1}$, Zhen Liu $^{1}$, Hong Jiang ${ }^{1,2,3}$, \\ Beisha Tang ${ }^{1,2,3}$ and Junling Wang ${ }^{1,2,3}$
}

A young Chinese male patient was identified as homozygous for Machado-Joseph disease (MJD)/spinocerebellar ataxia type 3. This patient had a 4-year medical history mainly presenting severe ataxia, abnormal eye movement and pyramidal signs. Magnetic resonance imaging of the brain and cervical spinal cord revealed no obvious abnormality despite the severe symptoms and the findings of an electromyogram. However, brainstem auditory evoked potential indicated peripheral impairment and visual evoked potential indicated central impairment of his visual pathways. Molecular diagnosis revealed the pattern of CAG repeat units of this patient was 71/71. This case demonstrates that homozygosity for MJD enhances the clinical severity of the disease, which suggests that genetic education is of great importance.

Journal of Human Genetics (2015) 60, 157-160; doi:10.1038/jhg.2014.117; published online 8 January 2015

\section{INTRODUCTION}

Machado-Joseph disease (MJD)/spinocerebellar ataxia (SCA) type 3 (SCA3; OMIM\#109150) is the most common form of SCAs worldwide and is an autosomal dominant neurodegenerative disorder. Its causative mutation is unstable CAG repeat expansions in exon 10 of the ATXN3 gene, which is located at $14 \mathrm{q} 32.1 .^{1} \mathrm{MJD} / \mathrm{SCA} 3$ is characterized by cerebellar ataxia, pyramidal signs and progressive external ophthalmoplegia. Other clinical features include dystonia, muscle rigidity, bradykinesia and peripheral amyotrophy. In homozygous cases, the disease causing CAG repeat expansion is present in both alleles of the gene. Here, we report a case of an early-onset MJD/SCA3 male patient from the Chinese mainland with CAG repeat sizes of $71 / 71$.

\section{CASE REPORT}

This patient gradually developed gait disturbances and slurred speech since the age of 18 . Four years later, when he first sought medical advice, he exhibited a spastic gait and difficulty walking that was accompanied by clumsiness of the upper limbs, and his right side was more severely affected than his left. Moreover, he also exhibited slurred speech and dysphagia. Neurological examination revealed mild gaze-evoked horizontal nystagmus, mild saccade hypermetria and mild hearing loss. Muscle tone was increased in all the four extremities, whereas muscle strength and volume were well preserved. Deep tendon reflexes in the knee were brisk. The patient performed poorly in the finger-nose test and presented with heel-kneel-shin ataxia. He was positive for ankle clonus and Babinski's sign bilaterally.

This patient was the product of consanguineous marriages, and his parents were first cousins (Figure 1a). His maternal grandmother and paternal grandmother were sisters and both gradually developed motor disabilities late in life, but their detailed genetic statuses were unknown. His mother was 46 years old when she began to feel clumsy in walking. Neurological examination revealed slightly abnormal eye movement and poor tests of coordinated movement. Neurological examination of his father who was 48 years old revealed mild oculomotor disorders although having no rational symptoms. (Clinical and genetic data of affected members in this pedigree were summarized in Table 1.)

The results of routine blood and urine tests of the patient were normal. Electromyogram detected no unusual findings. Brainstem auditory evoked potential indicated peripheral impairment without exclusion of central impairment. Visual evoked potential results indicated central impairment of his visual pathways. There was no evidence of atrophy of the cerebellum or the brain stem on magnetic resonance imaging (MRI) (Figure 1d).

After genetic counseling, informed consent was obtained from affected members of this family, as approved by the Ethical Committee of Xiangya Hospital of the Central South University in China (equivalent to an Institutional Review Board). Combinational strategy of denaturing polyacrylamide gel electrophoresis with capillary electrophoresis revealed the pattern of CAG repeat units of this patient was 71/71 (Figures $1 \mathrm{~b}$ and e). Direct sequencing of PCR product indicated the detailed information about the trinucleotide repeat unit (Figure 1c).

The CAG repeat sizes of his mother and father were 71/15 and $71 / 27$, respectively. The patient had a sibling who was a presymptomatic homozygote for MJD/SCA3 (71/71). CAG repeat sizes of other related family members were in Supplementary Data.

\footnotetext{
${ }^{1}$ Department of Neurology, Xiangya Hospital, Central South University, Changsha, Hunan, P.R. China; ${ }^{2}$ Key Laboratory of Hunan Province in Neurodegenerative Disorders, Changsha, Hunan, P.R. China and ${ }^{3}$ State Key Laboratory of Medical Genetics, Changsha, Hunan, P.R. China 
a

I

II

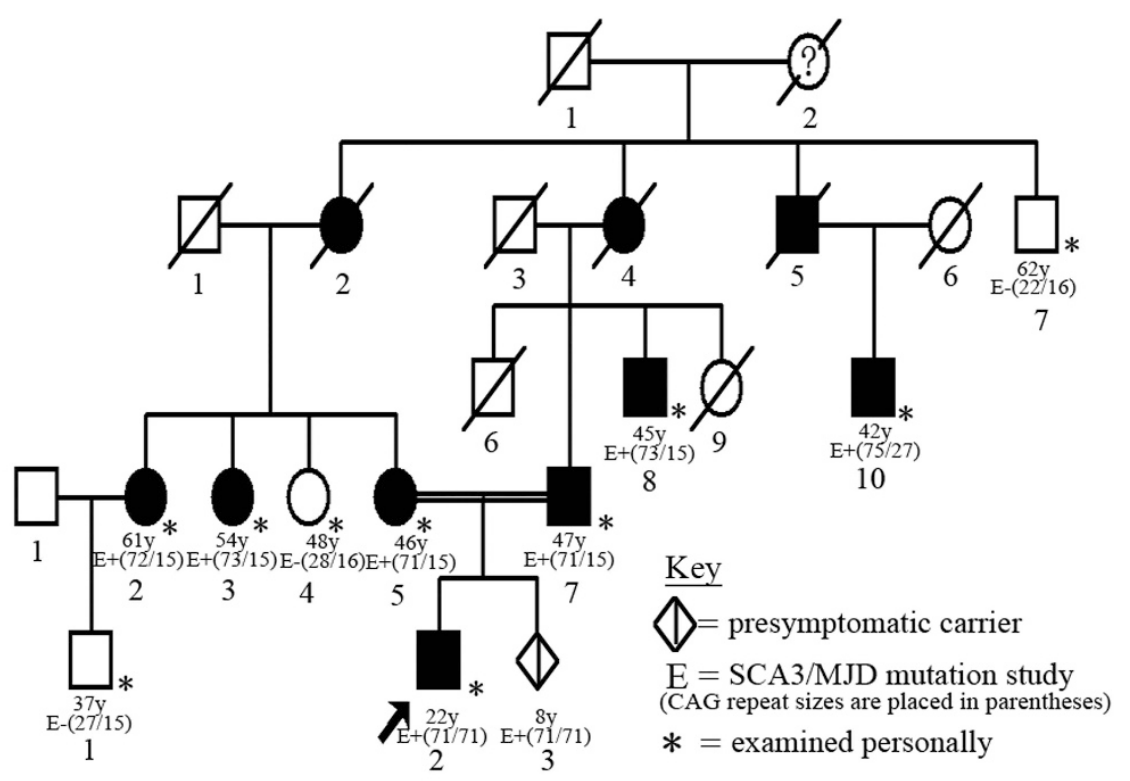

d

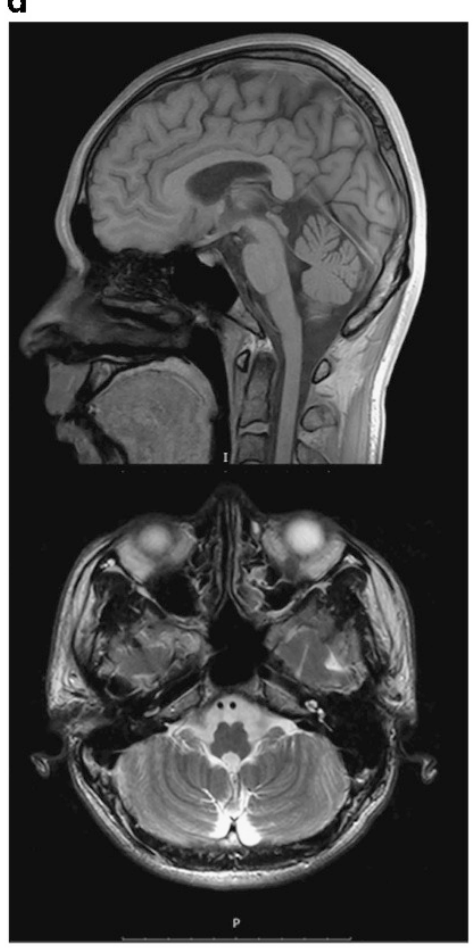

e

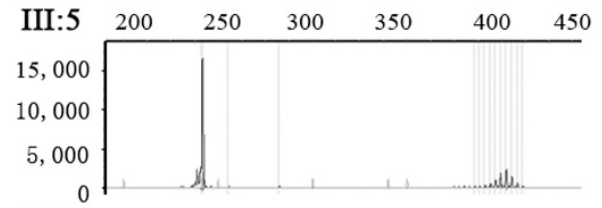

III:7

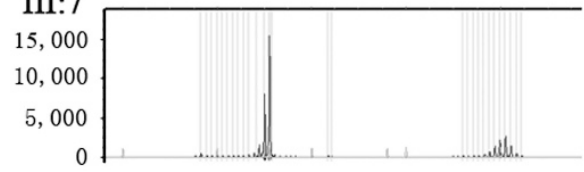

IV:2
15,000
10,000
5,000
0

IV:3
10,000
5,000
0

Figure 1 (a) Patient pedigree (to protect the privacy of presymptomatic affected individuals, the diamond instead). (b) Electropherogram of $8 \%$ denaturing polyacrylamide gel electrophoresis. Marker: pUC19 DNA/Mspl (Hpall) Marker; BC, blank control; NC, negative control; PC, positive control. (c) Electropherogram of direct sequencing of PCR product from the proband: CAG repeat units from 237 to $449 \mathrm{bp}$. (PCR products were cloned to T-Vector pMD19 (Simple) followed by sequencing of two randomly chosen clones). (d) Cranial magnetic resonance images (upper: sagittal T1 (TR/TE=212.0/5.2 ms); lower: axial T2 $(T R / T E=4500 / 95.0 \mathrm{~ms})$ ) of the MJD/SCA3 homozygous patient from China were normal at 22 years old. (e) Electropherogram of the capillary electrophoresis. From top to bottom: III:5(mother), III:7(father), IV:2(proband) and IV:3(sibling). A full color version of this figure is available at the Journal of Human Genetics journal online.

\section{DISCUSSION}

Homozygous MJD/SCA3 patients are rare, and most may be the products of consanguineous marriages. Before this report, nine pedigrees have been reported as the first description of a homozygous MJD/SCA3 case. $^{2-8}$ In addition to the characteristic symptoms that include cerebellar ataxia, pyramidal signs and extrapyramidal syndrome, non-characteristic features, such as spastic paraplegia phenotypes, cognitive, psychiatric disturbances and sleep disorders have also been observed.

As the first MJD/SCA3 homozygote case reported in mainland China, our patient's early age of onset and prominent neurologic impairments were distinct from the symptoms exhibited by his 
Table 1 Summary of clinical and genetic data of affected members in this family

\begin{tabular}{|c|c|c|c|c|c|c|c|}
\hline $\begin{array}{l}\text { Patient } \\
\text { ID }\end{array}$ & Gender & $\begin{array}{l}\text { Onset } \\
\text { age } \\
\text { (years) }\end{array}$ & $\begin{array}{l}\text { Current } \\
\text { age } \\
\text { (years) }\end{array}$ & $\begin{array}{l}C A G \\
\text { repeat } \\
\text { unit }\end{array}$ & Clinical features & $\begin{array}{l}\text { ICARS score (posture and gait disturbances, } \\
\text { kinetic functions, speech disorders and } \\
\text { oculormotor disorders) }\end{array}$ & $\begin{array}{l}\text { SARA } \\
\text { score }\end{array}$ \\
\hline$|I|: 2$ & Female & 55 & 61 & $72 / 15$ & $\begin{array}{l}\text { Wheel chaired, bradykinesia, nearly blind in both the eyes (cataract), } \\
\text { severe dysarthria, mild dysphagia, hypotonia and hyporeflexia }\end{array}$ & Completing impossible & \\
\hline III:3 & Female & 50 & 54 & $73 / 15$ & $\begin{array}{l}\text { Horizontal gaze-evoked nystagmus, diplopia, calculation and memory } \\
\text { deterioration, dizzy, moderate dysarthria, mild dysphagia, weakness in } \\
\text { lower limbs and ataxic gait }\end{array}$ & $44 / 100(18 / 34,20 / 52,2 / 8$ and $4 / 6)$ & $14 / 40$ \\
\hline III:5 & Female & 46 & 46 & $71 / 15$ & $\begin{array}{l}\text { Slightly impaired pursuit, slightly enlarged spread of feet and slow gait } \\
\text { speed, unable to walk with feet in tandem position }\end{array}$ & $18 / 100(7 / 34,10 / 52,0 / 8$ and $1 / 6)$ & $7 / 40$ \\
\hline III:7 & Male & 47 & 47 & $71 / 27$ & $\begin{array}{l}\text { Horizontal gaze-evoked nystagmus, slightly impaired pursuit and unable } \\
\text { to walk with feet in tandem position }\end{array}$ & $10 / 100(3 / 34,4 / 52,0 / 8$ and $3 / 6)$ & $5 / 40$ \\
\hline III:8 & Male & 48 & 48 & $73 / 15$ & $\begin{array}{l}\text { Horizontal gaze-evoked nystagmus, unable to walk with feet in tandem } \\
\text { position }\end{array}$ & $8 / 100(3 / 34,4 / 52,0 / 8$ and $1 / 6)$ & $5 / 40$ \\
\hline III:10 & Male & 57 & 62 & $75 / 27$ & $\begin{array}{l}\text { Slow saccades, horizontal gaze-evoked nystagmus, severe dysarthria, } \\
\text { mild dysphagia, weakness, hypertonia, hypereflexia and spastic gait } \\
\text { disturbance }\end{array}$ & $47 / 100(20 / 34,20 / 52,3 / 8$ and $4 / 6)$ & $18 / 40$ \\
\hline IV:2 & Male & 18 & 22 & $71 / 71$ & $\begin{array}{l}\text { Gaze-evoked horizontal nystagmus, dysarthria, dysphagia, hyperreflexia, } \\
\text { Babinski's sign, clonus, spastic gait disturbance and spasticity of the } \\
\text { lower limbs }\end{array}$ & $51 / 100(27 / 34,19 / 52,4 / 8$ and $1 / 6)$ & $29 / 40$ \\
\hline
\end{tabular}

Abbreviations: ICARS, International Cooperation Ataxia Rating Scale; SARA, Scale for Assessment and Rating of Ataxia.

parents. The inverse correlation between the sizes of the CAG repeats and the age of onset is widely recognized. The normal ATXN3 allele, $A P O E \varepsilon 2$ allele and gene dosage from the evidence of homozygotes could also influence the age of onset of MJD/SCA3. Three cases, including ours, presented with severe symptoms without evidence of cerebellar or brain stem atrophy on MRI. The results of a few reports have suggested that the severity of imaging findings is associated with multiple factors such as the number of CAG repeats, age, disease stage and so on. ${ }^{9,10}$ MRI findings may be mild at certain stages of MJD despite severe clinical findings. ${ }^{11}$ The younger onset age and relatively shorter disease duration may accounts for the mismatch between the relatively reserved cerebellum and brain stem on MRI and clinical severity. The characteristic of homozygotes indicates that imaging tests are neither ideal methods for early diagnosis nor main indicators of clinical severity. Thus, regular follow-ups should be performed, and synthetic judgments based on the molecular diagnosis, clinical condition and related diagnostic tests should be made. So, we have established the routine molecular diagnosis procedures of SCA with CAG repeat expansion, namely initial screening with $8 \%$ denaturing polyacrylamide gel electrophoresis followed by capillary electrophoresis identifying CAG repeat sizes. Finally, direct sequencing of PCR product reveals the detailed information of the target sequence.

Another interesting phenomenon existing in our case is the sizes of expanded CAG repeats of the expansion carriers in this family were uniform (71 repeats). Repeat instability during transmission is an important character of SCAs. A new study revealed all conditions of expansion, contraction and stability during transmission can occur with $29.7 \%$ stable transmission in MJD/SCA3. ${ }^{12}$ Stable transmission is rare after several generational transmissions in MJD/SCA3 in our case. The mechanisms of repeat instability are complicated and still unclear. $^{13}$

Unfortunately, no medication has been proven to slow the course of this disease. Molecular diagnoses have made it possible to detect the genetic statuses of at-risk family members of affected individuals. Genetic counseling is necessary to allow affected families to make informed medical and personal decisions. The importance of avoiding consanguineous marriages is paramount; thus, genetic education is of great importance.

\section{CONFLICT OF INTEREST}

The authors declare that they have no conflict of interest.

\section{ACKNOWLEDGEMENTS}

This work was funded by the Program of National Natural Science Foundation of China (\#81130021, 81300981, 81250015), National Department Public Benefit Research Foundation (\#201302001), Key Projects in the National Science \& Technology Pillar Program during the Twelfth Five-year Plan Period (\#2012BAI09B04). Fundamental Research Funds for the Central Universities (\#2012QNZT107) and Grant from the Research Fusnd for the Doctoral Program of Higher Education of China (\#20120162120048). We thank Professor Chris Jones very much for polishing our paper.

1 Kawaguchi, Y., Okamoto, T., Taniwaki, M., Aizawa, M., Inoue, M., Katayama, S. et al. CAG expansions in a novel gene for Machado-Joseph disease at chromosome 14q32.1. Nat. Genet. 8, 221-228 (1994).

2 Coutinho, P., Guimaraes, A. \& Scaravilli, F. The pathology of Machado-Joseph disease. Report of a possible homozygous case. Acta Neuropathol. 58, 48-54 (1982).

3 Lang, A. E., Rogaeva, E. A., Tsuda, T., Hutterer, J. \& St George-Hyslop, P. Homozygous inheritance of the Machado-Joseph disease gene. Ann. Neurol. 36, 443-447 (1994).

4 Lerer, I., Merims, D., Abeliovich, D., Zlotogora, J. \& Gadoth, N. Machado-Joseph disease: correlation between the clinical features, the CAG repeat length and homozygosity for the mutation. Eur. J. Hum. Genet. 4, 3-7 (1996).

5 Sobue, G., Doyu, M., Nakao, N., Shimada, N., Mitsuma, T., Maruyama, H. et al. Homozygosity for Machado-Joseph disease gene enhances phenotypic severity. J. Neurol. Neurosurg. Psychiatry 60, 354-356 (1996).

6 Fukutake, T., Shinotoh, H., Nishino, H., Ichikawa, Y., Goto, J., Kanazawa, I. et al. Homozygous Machado-Joseph disease presenting as REM sleep behaviour disorder and prominent psychiatric symptoms. Eur. J. Neurol. 9, 97-100 (2002).

7 Carvalho, D. R., La Rocque-Ferreira, A., Rizzo, I. M., Imamura, E. U. \& Speck-Martins, C. E. Homozygosity enhances severity in spinocerebellar ataxia type 3. Pediatr. Neurol. 38, 296-299 (2008).

8 Lysenko, L., Grewal, R. P., Ma, W. \& Peddareddygari, L. R. Homozygous Machado Joseph Disease: a case report and review of literature. Can. J. Neurol. Sci. 37, 521-523 (2010).

9 Abe, Y., Tanaka, F., Matsumoto, M., Doyu, M., Hirayama, M., Kachi, T. et al. CAG repeat number correlates with the rate of brainstem and cerebellar atrophy in Machado-Joseph disease. Neurology 51, 882-884 (1998). 
10 Camargos, S. T., Marques, W. Jr. \& Santos, A. C. Brain stem and cerebellum volumetric analysis of Machado Joseph disease patients. Arq Neuropsiquiatr. 69 292-296 (2011).

11 Tokumaru, A.M., Kamakura, K., Maki, T., Murayama, S., Sakata, I., Kaji, T. et al. Magnetic resonance imaging findings of Machado-Joseph disease: histopathologic correlation. J. Comput. Assist. Tomogr. 27, 241-248 (2003).
12 Martins, S., Pearson, C. E., Coutinho, P., Provost, S., Amorim, A., Dube, M. P. et al Modifiers of (CAG)n instability in Machado-Joseph disease (MJD/SCA3) transmissions: an association study with DNA replication, repair and recombination genes. Hum. Genet. 133, 1311-1318 (2014).

13 Pearson, C. E., Nichol Edamura, K. \& Cleary, J. D. Repeat instability: mechanisms of dynamic mutations. Nat. Rev. Genet. 6, 729-742 (2005)

Supplementary Information accompanies the paper on Journal of Human Genetics website (http://www.nature.com/jhg) 